\title{
Surgical approach for the treatment of thymic carcinoma: 201 cases from a multi-institutional study
}

\section{Yeong Jeong Jeon ${ }^{1}$, Yong Soo $\mathrm{Choi}^{1}$, Jong Ho Cho ${ }^{1}$, Hong Kwan Kim ${ }^{1}$, Geun Dong Lee ${ }^{2}$, Dong Kwan Kim², Chang Hyun Kang ${ }^{3}$, Young Tae Kim³ ${ }^{3}$ Chang Young Lee ${ }^{4}$, Jin Gu Lee ${ }^{4}$, other members of the Korean Association for Research on the Thymus (KART)}

\author{
${ }^{1}$ Department of Thoracic and Cardiovascular Surgery, Samsung Medical Center, Sungkyunkwan University School of Medicine, \\ Seoul, Korea \\ ${ }^{2}$ Department of Thoracic and Cardiovascular Surgery, Asan Medical Center, University of Ulsan College of Medicine, Seoul, Korea \\ ${ }^{3}$ Department of Thoracic and Cardiovascular Surgery, Seoul National University Hospital, Seoul National University College of \\ Medicine, Seoul, Korea \\ ${ }^{4}$ Department of Thoracic and Cardiovascular Surgery, Severance Hospital, Yonsei University College of Medicine, Seoul, Korea
}

Received: November 11, 2020

Revised: November 26, 2020

Accepted: November 26, 2020

Corresponding author:

Yong Soo Choi

Department of Thoracic and Cardiovascular Surgery, Samsung Medical Center, Sungkyunkwan University School of Medicine, 81 Irwon-ro, Gangnam-gu, Seoul 06351, Korea

Tel: +82-2-3410-1696

E-mail: choi.smcts@gmail.com

This is an Open Access article distributed under the terms of the Creative Commons Attribution Non-Commercial License (https:// creativecommons.org/licenses/ by-nc/4.0/).

\section{ABSTRACT}

Purpose: This study aimed to compare the outcomes of surgical approach (video-assisted thoracoscopic surgery [VATS] vs. sternotomy vs. thoracotomy) for the treatment of thymic carcinoma

Methods: We retrospectively reviewed 201 patients with pathologically proven thymic carcinoma who underwent surgical resection at four Korean institutions.

Results: From 2007 to 2013, 158 sternotomy, 33 VATS and 10 thoracotomy were conducted for thymic carcinoma. Open group underwent more preoperative biopsy $(41.8 \%$ and $50 \%$ vs. $15.2 \%, P=0.012)$ and neoadjuvant treatment $(22.2 \%$ and $30 \%$ vs. $0 \%$, $\mathrm{P}=0.008$ ) than VATS group. In preoperative imaging, tumor size of VATS group was smaller than sternotomy group $(3.8 \pm 1.1 \mathrm{~cm}$ vs. $5.8 \pm 2 \mathrm{~cm}, \mathrm{P}<0.05)$ and $91 \%$ of the VATS group was clinical tumor-node-metastasis (TNM) stage I. The lengths of chest tube and mechanical ventilation duration, postoperative hospital day and intensive care unit stay were shorter in VATS group than open group $(P<0.001)$. The incidence of postoperative complications of VATS group was lower than sternotomy group $(P=0.014)$. The 5 -year overall survival of the sternotomy, VATS and thoracotomy group were $100 \%$, $100 \%$ and $87.5 \% \pm 11.7 \%$, respectively $(P=0.107)$. The 5 -year recurrence-free survival rate was not significantly different between the groups $(55.4 \% \pm 4.5 \%, 67.9 \% \pm 12.1 \%$, and $87.5 \% \pm 11.7 \% ; P=0.131$ )

Conclusion: The VATS approach of surgical treatment for thymic carcinoma can be selectively employed in small $(<5 \mathrm{~cm})$ and TNM stage I tumor without compromise of oncologic outcome.

Keywords: Thymus neoplasms; Carcinoma; Thoracic surgery, video-assisted 


\section{INTRODUCTION}

Thymic carcinoma is malignant tumor originating from the thymic epithelium, and it represents a very heterogeneous group of lesions with a wide spectrum of morphologic and prognostic features [1]. Thymic carcinoma is very rare, and only several studies on prognostic factors and treatments have been reported so far. According to most studies, surgical resection is the mainstay of treatment for thymic carcinoma [2-4].

Conventional transsternal approach has long been accepted as standard method of treatment for thymoma and thymic carcinoma. However, device developments and technological advances led to successful application of the minimally invasive approach for thymoma. Recently, minimally invasive procedure such as video-assisted thoracoscopic surgery (VATS) has been performed more frequently for resection of early-stage thymoma. Many literatures have reported the oncologic outcomes of VATS thymectomy for the treatment of thymoma. They showed that VATS thymectomy had less postoperative pain, the length of hospitalization and favorable oncologic outcomes [5-10]. In spite of the advantage of minimally invasive approach, the application of VATS for early-stage thymic carcinoma has controversy because of its poor prognosis and the lack of long-term data.

The present study was aimed to compare the outcomes of surgical approach (VATS vs. sternotomy vs. thoracotomy) for the treatment of thymic carcinoma.

\section{METHODS}

\section{Study cohort}

Korean Association for Research on the Thymus (KART) developed a multi-institutional database in 2014, and retrospectively collected the data of 1,663 patients with thymic epithelial tumor who underwent surgical treatment and biopsy between 2000 and 2013 at four Korean institutions. The KART database included patient characteristics, preoperative and pathologic tumor size, preoperative and final pathological Masaoka-Koga stage, pathological World Health Organization (WHO) classification, histologic type, type of resection, resection status, perioperative therapies, treatment, pattern of recurrence, and survival.

A total of 256 patients with pathologically proven thymic carcinoma underwent surgery. Among them, 201 patients underwent thymectomy via sternotomy $(n=158)$, VATS $(n=$ $33)$, or thoracotomy $(n=10)$. Patients who underwent diag- nostic $(n=17)$ or debulking surgery $(n=8)$, operation for recurrence $(n=16)$ and operation via other approach including transcervical, clamshell and sternotomy combined with thoracotomy $(n=14)$ were excluded. We were classified histologic type according to latest WHO classification and thymic neuroendocrine tumors and type B3 thymomas were excluded.

This study was approved by Institutional Review Board of Samsung Medical Center (2017-03-006). Informed consent was waived by the board.

\section{Statistical analysis}

Continuous data are described by mean \pm standard deviation and categoric data by frequencies and percentages. Comparison of preoperative baseline characteristics between three groups was analyzed by Kruskal-Wallis test for continuous variables, and the Pearson's chi-square and Fisher's exact tests for categorical variables, when appropriate.

The Kaplan-Meier method was used to analyze overall survival (OS) and recurrence-free survival (RFS). The log-rank test was used to assess the differences between survival rates. Univariate and multivariate Cox regression analyses were employed to evaluated OS and RFS prognostic factors. Chi-square test and Fisher's exact test, when appropriate, were used to evaluate the difference between groups. The statistical analysis was performed using SAS version 9.4 (SAS Institute Inc., Cary, NC, USA).

\section{RESULTS}

\section{Patient characteristics}

From January 2000 to December 2013, a total of 201 patients (158 sternotomy, 33 VATS, and 10 thoracotomy) were included in this study. Sex, age, smoking history, symptom, performance status, pulmonary function, body mass index, and comorbidity were not different between the groups. Open group including sternotomy and thoracotomy underwent preoperative biopsy and neoadjuvant treatment more frequently than VATS group. Baseline patient characteristics are summarized in Table 1.

\section{Factors associated with undergoing VATS}

Patients who underwent VATS had tumor less than $5 \mathrm{~cm}$ $(P<0.001)$ and clinical tumor-node-metastasis (TNM) stage I International Association for the Study of Lung Cancer/International Thymic Malignancies Interest Group (IASLC/ITMIG) 8th edition ( $P=0.001)$. Furthermore, tumor without vascular invasion on preoperative computed tomography (CT) were 
PRECISION AND FUTURE MEDICINE

Yeong Jeong Jeon, et al.

Table 1. Patient characteristics

\begin{tabular}{|c|c|c|c|c|}
\hline Characteristic & Sternotomy $(n=158)$ & VATS $(n=33)$ & Thoracotomy $(n=10)$ & P-value \\
\hline Male sex & $109(69)$ & $19(57.6)$ & $7(70)$ & 0.433 \\
\hline Age (yr) & $57.7 \pm 10.6$ & $60 \pm 11.7$ & $59.2 \pm 8.1$ & 0.497 \\
\hline Smoking history & & & & 0.551 \\
\hline None & $73(46.2)$ & $20(60.6)$ & $5(50)$ & \\
\hline Ex-smoker & $48(30.4)$ & $8(24.2)$ & $4(40)$ & \\
\hline Current smoker & $37(23.4)$ & $5(15.2)$ & $1(10)$ & \\
\hline Symptom & & & & 0.188 \\
\hline None & $89(56.3)$ & $25(75.8)$ & $5(50)$ & \\
\hline Chest pain & $43(27.2)$ & $3(9.1)$ & $2(20)$ & \\
\hline Cough & $13(8.2)$ & $2(6.1)$ & $1(10)$ & \\
\hline Face/arm swelling & $3(1.9)$ & 0 & 0 & \\
\hline Dyspnea & $9(5.7)$ & $2(6.1)$ & $2(20)$ & \\
\hline ECOG & & & & 0.283 \\
\hline 0 & $127(80.9)$ & $28(84.8)$ & $7(70)$ & \\
\hline 1 & $29(18.5)$ & $4(12.1)$ & $3(30)$ & \\
\hline 2 & $1(0.6)$ & 0 & 0 & \\
\hline 3 or 4 & 0 & $1(3)$ & 0 & \\
\hline FEV1 (\% predicted) & & & & 0.557 \\
\hline$\geq 80$ & $110(81.5)$ & $21(91.3)$ & $7(77.8)$ & \\
\hline$<80$ & $25(18.5)$ & $2(8.7)$ & $2(22.2)$ & \\
\hline DLCO (\% predicted) & & & & 0.847 \\
\hline$\geq 80$ & $62(73.8)$ & $10(71.4)$ & $5(62.5)$ & \\
\hline$<80$ & $22(26.2)$ & $4(28.6)$ & $3(37.5)$ & \\
\hline $\mathrm{BMI}\left(\mathrm{kg} / \mathrm{m}^{2}\right)$ & $24 \pm 3.1$ & $23.8 \pm 1.8$ & $25.2 \pm 2.7$ & 0.338 \\
\hline \multicolumn{5}{|l|}{ Comorbidity } \\
\hline Diabetes & $19(12)$ & $3(9.1)$ & $1(10)$ & 0.911 \\
\hline Hypertension & $52(32.9)$ & $9(27.3)$ & $5(5)$ & 0.407 \\
\hline Chronic obstructive pulmonary disease & $6(3.8)$ & $1(3)$ & 0 & 1.000 \\
\hline Autoimmune disease & $1(0.6)$ & $1(3)$ & 0 & 0.383 \\
\hline Preoperative biopsy & $66(41.8)$ & $5(15.2)$ & $5(50)$ & 0.012 \\
\hline Neoadjuvant treatment & $35(22.2)$ & 0 & $3(30)$ & 0.008 \\
\hline Concurrent chemoradiation & $5(3.2)$ & 0 & 0 & \\
\hline Chemotherapy & $28(17.7)$ & 0 & $3(30)$ & \\
\hline Radiotherapy & $2(1.3)$ & 0 & 0 & \\
\hline Response of neoadjuvant treatment & & & & 0.031 \\
\hline Partial response & $21(77.8)$ & 0 & $2(66.7)$ & \\
\hline Stable disease & $6(3.8)$ & 0 & $1(33.3)$ & \\
\hline
\end{tabular}

Values are presented as number (\%) or mean \pm standard deviation.

VATS, video-assisted thoracoscopic surgery; ECOG, European Cooperative Oncology Group; FEV1, forced expiratory volume in 1 second; DLCO, diffusing capacity of the lung for carbon monoxide; BMI, body mass index. 
PRECISION AND FUTURE MIEDICINE

Surgical treatment of thymic carcinoma

Table 2. Operative findings, procedures and pathologic findings

\begin{tabular}{|c|c|c|c|c|}
\hline Variable & Sternotomy $(n=158)$ & VATS $(n=33)$ & Thoracotomy $(n=10)$ & P-value \\
\hline Extent & & & & $<0.001$ \\
\hline Thymomectomy & $17(10.8)$ & $19(57.6)$ & $5(50)$ & \\
\hline Partial thymectomy & $7(4.4)$ & $7(21.2)$ & $1(10)$ & \\
\hline Total thymectomy & $134(84.8)$ & $7(21.2)$ & $4(40)$ & \\
\hline Tumor size & $6.4 \pm 2.3$ & $4.6 \pm 1.8$ & $3.9 \pm 2$ & $<0.001$ \\
\hline Lymph node dissection & $80(50.6)$ & $8(24.2)$ & $6(60)$ & 0.015 \\
\hline Additional procedure & $133(84.2)$ & $9(27.3)$ & $9(90)$ & $<0.001$ \\
\hline Lung, wedge resection & $92(58.2)$ & $5(15.2)$ & $5(50)$ & $<0.001$ \\
\hline Lung, segmentectomy & $2(1.3)$ & 0 & $1(10)$ & 0.209 \\
\hline Lung, lobectomy & $5(3.2)$ & $1(3)$ & $2(20)$ & 0.079 \\
\hline Diaphragm, resection & $2(1.3)$ & 0 & $1(10)$ & 0.209 \\
\hline Pericardium, resection & $80(50.6)$ & $4(12.1)$ & $7(70)$ & $<0.001$ \\
\hline Innominate vein, resection & $46(29.1)$ & 0 & $2(20)$ & $<0.001$ \\
\hline Phrenic nerve, resection & $45(28.5)$ & $2(6.1)$ & $4(40)$ & 0.015 \\
\hline Operation time (min) & $235(115-736)$ & $147(99-196)$ & $356(305-408)$ & $<0.001$ \\
\hline Blood loss & $350(22-8,000)$ & $135(50-22)$ & $825(450-1,200)$ & $<0.001$ \\
\hline Transfusion & $39(24.8)$ & 0 & $3(30)$ & 0.005 \\
\hline Pathologic TNM stage & & & & $<0.001$ \\
\hline I & $57(36.1)$ & $27(81.8)$ & $2(20)$ & \\
\hline$\|$ & $10(6.3)$ & $1(3)$ & $1(10)$ & \\
\hline IIIA & $43(27.2)$ & 0 & $2(20)$ & \\
\hline IIIB & $11(7)$ & $1(3)$ & 0 & \\
\hline IVA & $20(12.7)$ & $2(6.1)$ & $4(40)$ & \\
\hline IVB & $17(10.8)$ & $2(6.1)$ & $1(10)$ & \\
\hline Subtype & & & & 0.861 \\
\hline Squamous cell carcinoma & $119(78.3)$ & $29(87.9)$ & $9(90)$ & \\
\hline Lymphoepithelioma-like & $6(3.9)$ & 0 & 0 & \\
\hline Sarcomatoid & $3(2)$ & 0 & 0 & \\
\hline Mucoepidermoid & $2(1.3)$ & 0 & 0 & \\
\hline Adenocarcinoma & $3(2)$ & 0 & 0 & \\
\hline Clear cell carcinoma & $1(0.7)$ & 0 & 0 & \\
\hline Not otherwise specified & $11(7.2)$ & $4(12.1)$ & 0 & \\
\hline Complete resection & & & & 0.216 \\
\hline RO & $128(81)$ & $32(97)$ & $7(70)$ & \\
\hline $\mathrm{R} 1$ & $29(18.4)$ & 0 & $3(30)$ & \\
\hline $\mathrm{R} 2$ & $1(0.6)$ & $1(3)$ & 0 & \\
\hline
\end{tabular}

Values are presented as number (\%) or median (range).

VATS, video-assisted thoracoscopic surgery; TNM tumor-node-metastasis.

subjected to VATS $(P=0.049)$. Tumor location, size, TNM stage and invasion of surrounding tissue on preoperative $\mathrm{CT}$ image are described in Supplementary Table 1.

\section{Surgical outcomes}

The operation time of VATS was shorter than sternotomy and thoracotomy $(P<0.001)$. The amount of blood loss and the 
PRECISION AND FUTURE MIEDICINE

Yeong Jeong Jeon, et al.

Table 3. Postoperative outcome

\begin{tabular}{lcccc}
\hline Variable & Sternotomy $(\mathbf{n}=158)$ & VATS $(\mathbf{n}=33)$ & Thoracotomy $(\mathbf{n}=10)$ & P-value \\
\hline Mortality at 30 days & $2(1.3)$ & 0 & 0 & 1.000 \\
Chest tube duration (day) & $5(2-13)$ & $2(1-7)$ & $1.5(0-3)$ & $<0.001$ \\
Ventilation duration (day) & $0(0-6)$ & 0 & $1(0-2)$ & $<0.001$ \\
Intensive care unit stay (day) & $1(0-44)$ & $0(0-42)$ & $0.5(0-9)$ & $<0.001$ \\
Hospital stay (day) & $9(3-63)$ & $4(2-158)$ & $7(4-19)$ & 0.350 \\
Adjuvant therapy & & & & $3(30)$ \\
None & $30(19)$ & $10(30.3)$ & $4(40)$ & \\
Chemotherapy & $35(22.2)$ & $4(12.1)$ & $3(30)$ & 0.014 \\
Radiotherapy & $73(46.2)$ & $15(45.5)$ & 0 & \\
Concurrent chemoradiation & $20(12.7)$ & $4(12.1)$ & 0 & \\
Complications & $44(27.8)$ & $3(9.1)$ & 0 & \\
Prolonged air leak (>5 days) & $1(0.6)$ & 0 & 0 & \\
Atelectasis & $2(1.3)$ & 0 & 0 & \\
Pneumonia & 0 & 0 & 0 & \\
Acute lung injury & $1(0.6)$ & 0 & 0 & \\
Pulmonary thromboembolism & $1(0.6)$ & 0 & 0 & \\
Re-intubation & $1(0.6)$ & 0 & 0 & \\
Chylothorax & $2(1.3)$ & $1(3)$ & 0 & \\
Arrhythmia & $8(5.1)$ & 0 & 0 & \\
Wound infection & $1(0.6)$ & 0 & 0 & \\
Bleeding & $2(1.3)$ & 0 & 0 & \\
Grade & $11(7)$ & 0 & 0 & \\
I & $21(13.3)$ & $1(3)$ & 0 & \\
II & $10(6.3)$ & 0.134 & \\
III & $2(1.3)$ & 0 & \\
IV & & 0 & \\
\hline
\end{tabular}

Values are presented as number (\%) or median (range ).

VATS, video-assisted thoracoscopic surgery.

incidence of transfusion were lower in the VATS group than in the open group $(P<0.001$ and $P=0.005)$.

In terms of extent of resection, most of the sternotomy group underwent total thymectomy $(P<0.001)$, and lymph node dissection was performed more frequently in open group $(P=0.015)$. Furthermore, additional procedures including resection of lung, pericardium, innominate vein and phrenic nerve were conducted more frequently in open group $(P<0.001)$. Complete resection was not different between the groups. Details are shown in Table 2.

One patient of sternotomy group was died within postoperative 30 days. The lengths of postoperative hospital stay (POHS), intensive care unit (ICU) stay and chest tube duration were shorter in the VATS group than in the open group $(\mathrm{P}<$
0.001). The incidence of postoperative complications of the VATS group was lower than those of open group $(P=0.014)$. Details of the incidence of complications are shown in Table 3.

\section{Survival and oncologic outcomes}

The median follow-up duration was 50.3 months. The 5-year OS rates of the sternotomy and VATS group were $100 \%$, while thoracotomy group was $87.5 \% \pm 11.7 \%(P=0.107)$. The 5 -year RFS rates were not significantly different between the groups (55.4\% $\pm 4.5 \%$ for sternotomy group, $67.9 \% \pm 12.1 \%$ for VATS group, $32.8 \% \pm 18.3 \%$ for the thoracotomy group, $\mathrm{P}=0.131$ ). These graphs are presented in Fig. 1.

In terms of recurrence patterns, local recurrence was not different between the groups, while the thoracotomy group 

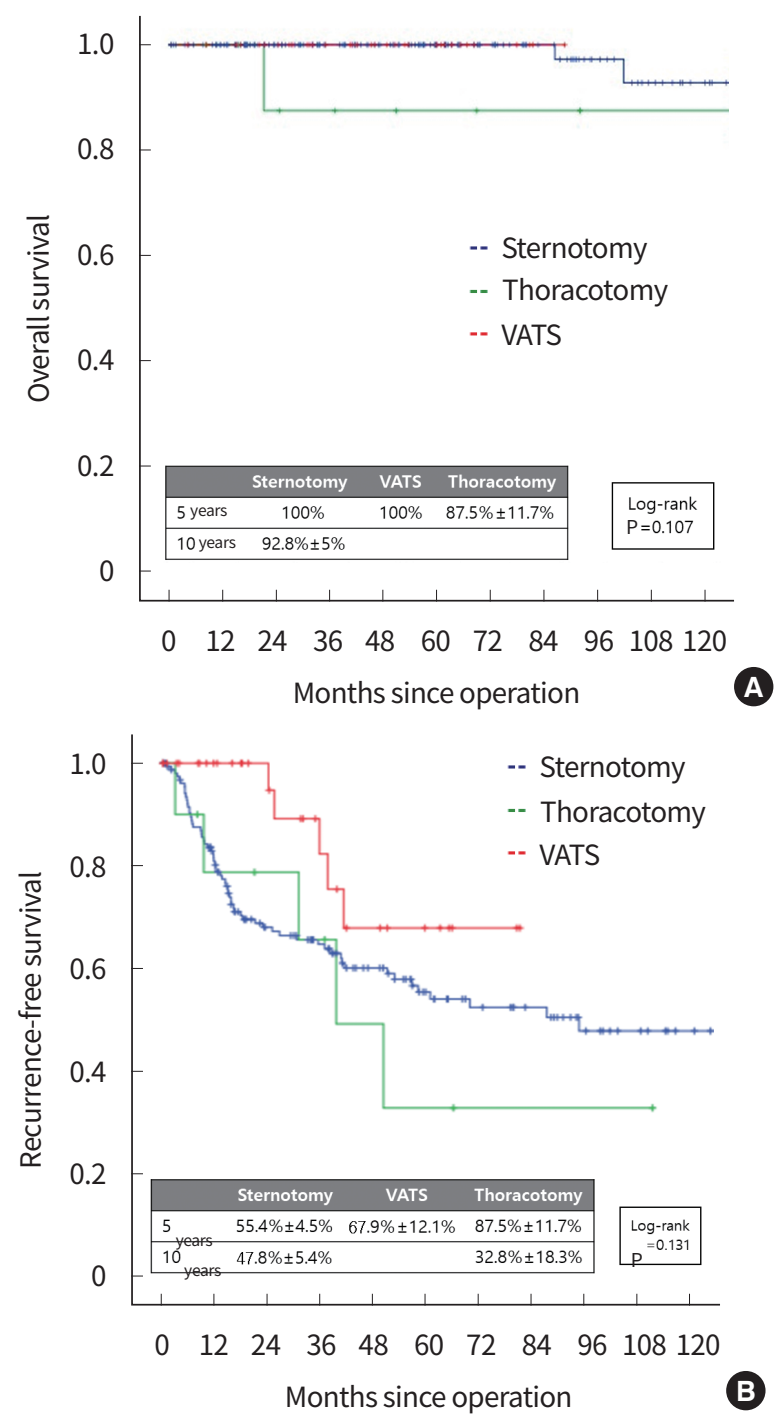

Fig. 1. Overall survival (A) and recurrence-free survival (B) of 201 patients. VATS, video-assisted thoracoscopic surgery.

Table 4. Pattern of recurrence

\begin{tabular}{lcccc}
\hline Variable & $\begin{array}{c}\text { Sternotomy } \\
(n=158)\end{array}$ & $\begin{array}{c}\text { VATS } \\
(n=33)\end{array}$ & $\begin{array}{c}\text { Thoracotomy } \\
(n=10)\end{array}$ & P-value \\
\hline Site of recurrence & & & & \\
Local & $20(33.9)$ & $3(60)$ & $2(40)$ & 0.847 \\
Regional & $19(32.2)$ & $1(20)$ & $2(40)$ & \\
Distant & $20(33.9)$ & $1(20)$ & $1(20)$ & \\
\hline
\end{tabular}

Values are presented as number (\%).

VATS, video-assisted thoracoscopic surgery.

had more regional recurrence than the VATS group (30\% vs. $3 \%, P=0.04)$. Details are shown in Table 4.
Table 5. Postoperative outcome of patients with tumor less than 5 $\mathrm{cm}$ and TNM stage I

\begin{tabular}{|c|c|c|c|}
\hline Variable & $\begin{array}{l}\text { Sternotomy } \\
\qquad(n=28)\end{array}$ & $\begin{array}{l}\text { VATS } \\
(n=27)\end{array}$ & P-value \\
\hline Extent & & & $<0.001$ \\
\hline Thymomectomy & $2(7.1)$ & $16(59.3)$ & \\
\hline Partial thymectomy & 0 & $5(18.5)$ & \\
\hline Total thymectomy & $26(92.9)$ & $6(22.2)$ & \\
\hline Pathologic tumor size $(\mathrm{cm})$ & $4.7 \pm 1.7$ & $4.2 \pm 1.6$ & 0.182 \\
\hline Pathologic TNM stage & & & 0.013 \\
\hline I & $17(60.7)$ & $22(81.5)$ & \\
\hline II & $2(7.1)$ & 0 & \\
\hline IIIA & $6(21.4)$ & 0 & \\
\hline IIIB & 0 & $1(3.7)$ & \\
\hline IVA & $3(10.7)$ & $2(7.4)$ & \\
\hline IVB & 0 & $2(7.4)$ & \\
\hline Concomitant procedure & $21(75)$ & $7(25.9)$ & $<0.001$ \\
\hline Lung, wedge resection & $14(50)$ & $4(14.8)$ & 0.005 \\
\hline Lung, lobectomy & 0 & $1(3.7)$ & 1.000 \\
\hline Pericardium, resection & $12(42.9)$ & $3(11.1)$ & 0.008 \\
\hline Innominate vein, resection & $8(28.6)$ & 0 & 0.004 \\
\hline Phrenic nerve, resection & $3(10.7)$ & $2(7.4)$ & 1.000 \\
\hline Operation time (min) & $175(115-535)$ & $102(13-215)$ & $<0.001$ \\
\hline Blood loss (mL) & $375(0-2,000)$ & $5(0-400)$ & $<0.001$ \\
\hline Transfusion & $3(10.7)$ & 0 & 0.236 \\
\hline Complete resection & & & 0.491 \\
\hline Ro & $28(100)$ & $26(96.3)$ & \\
\hline $\mathrm{R} 1$ & 0 & 0 & \\
\hline $\mathrm{R} 2$ & 0 & $1(3.7)$ & \\
\hline Mortality at 30 days & 0 & 0 & 1.000 \\
\hline Chest tube duration (day) & $4(2-13)$ & $2(1-7)$ & $<0.001$ \\
\hline Ventilation duration (day) & $0(0-2)$ & 0 & 0.051 \\
\hline Intensive care unit stay (day) & $1(0-5)$ & $0(0-1)$ & $<0.001$ \\
\hline Hospital stay (day) & $8(3-20)$ & $4(2-13)$ & $<0.001$ \\
\hline Adjuvant therapy & & & 0.755 \\
\hline None & $7(25)$ & $10(37)$ & \\
\hline Chemotherapy & $4(14.3)$ & $3(11.1)$ & \\
\hline Radiotherapy & $14(50)$ & $10(37)$ & \\
\hline $\begin{array}{l}\text { Concurrent } \\
\text { chemoradiotherapy }\end{array}$ & $3(10.7)$ & $4(14.8)$ & \\
\hline
\end{tabular}

(Continued to the next page) 
Table 5. Continued

\begin{tabular}{|c|c|c|c|}
\hline Variable & $\begin{array}{l}\text { Sternotomy } \\
(n=28)\end{array}$ & $\begin{array}{l}\text { VATS } \\
(n=27)\end{array}$ & P-value \\
\hline Complications & $6(21.4)$ & $2(7.4)$ & 0.252 \\
\hline $\begin{array}{l}\text { Prolonged air leak } \\
\text { (>5 days })\end{array}$ & 0 & 0 & \\
\hline Atelectasis & 0 & 0 & \\
\hline Pneumonia & 0 & 0 & \\
\hline Acute lung injury & 0 & 0 & \\
\hline $\begin{array}{l}\text { Pulmonary } \\
\text { thromboembolism }\end{array}$ & 0 & 0 & \\
\hline Re-intubation & 1 & 1 & \\
\hline Chylothorax & 2 & 0 & \\
\hline Arrhythmia & 2 & 0 & \\
\hline Wound infection & 0 & 0 & \\
\hline Bleeding & 0 & 0 & \\
\hline Others & 1 & 1 & \\
\hline Grade & & & 0.296 \\
\hline 1 & 2 & 2 & \\
\hline ॥ & 3 & 0 & \\
\hline III & 1 & 0 & \\
\hline IV & 0 & 0 & \\
\hline
\end{tabular}

Values are presented as number (\%), mean \pm standard deviation, or median (range).

TNM, tumor-node-metastasis; VATS, video-assisted thoracoscopic surgery.

Table 6. Pattern of recurrence of patients with tumor less than 5 $\mathrm{cm}$ and TNM stage I

\begin{tabular}{lllc}
\hline Variable & $\begin{array}{c}\text { Sternotomy } \\
(\mathrm{n}=28)\end{array}$ & $\begin{array}{c}\text { VATS } \\
(\mathrm{n}=27)\end{array}$ & P-value \\
\hline $\begin{array}{l}\text { Site of recurrence } \\
\text { Local }\end{array}$ & $2(7.1)$ & $3(11.1)$ & 0.38 \\
Regional & $1(3.6)$ & $1(3.7)$ & \\
Distant & $5(17.9)$ & $1(3.7)$ &
\end{tabular}

Values are presented as number (\%).

TNM, tumor-node-metastasis; VATS, video-assisted thoracoscopic surgery.

\section{Subgroup analysis for tumor less than $5 \mathrm{~cm}$ and clini- cal TNM stage I}

A total of 55 patients (28 sternotomy and 27 VATS) had tumor less than $5 \mathrm{~cm}$ and clinical TNM stage I. The lengths of POHS, ICU stay and chest tube duration were shorter in the VATS group than in the sternotomy group $(P<0.001)$. The incidence of postoperative complications of the VATS group did
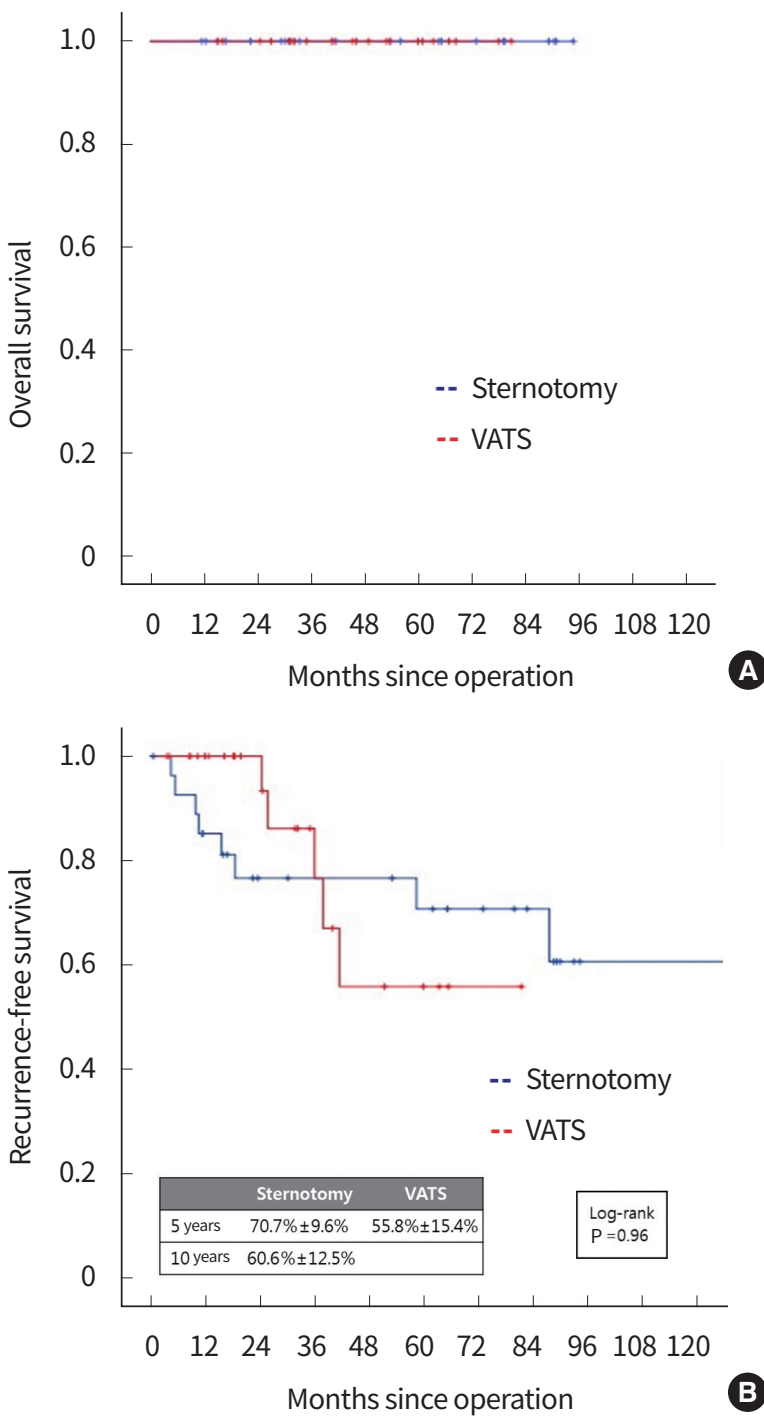

Fig. 2. Overall survival (A) and recurrence-free survival (B) of patients with tumor less than $5 \mathrm{~cm}$ and tumor-node-metastasis (TNM) stage I. VATS, video-assisted thoracoscopic surgery.

not differ from the sternotomy $(P=0.252)$. Details are described in Table 5.

The 5 -year OS rates of subgroup were $100 \%$ in both groups. The 5-year RFS rates of subgroup were not significantly different between the groups $(70.7 \% \pm 9.6 \%$ for sternotomy group, $55.8 \% \pm 15.4 \%$ for VATS group, $P=0.960$ ). These graphs are illustrated in Fig. 2. The pattern of recurrence of subgroup was not different between the groups (Table 6).

\section{Predictors of OS and RFS}

The predictors of OS and RFS identified by univariable and multivariable analysis are listed in Tables 7, 8. In multivariable Cox regression analysis of prognostic factors, surgical approach was not significantly associated with OS $(P=$ 
PRECISION AND FUTURE MIEDICINE

Surgical treatment of thymic carcinoma

Table 7. Cox regression analysis of prognostic factors of overall survival

\begin{tabular}{|c|c|c|c|c|c|c|}
\hline \multirow{2}{*}{ Variable } & \multicolumn{3}{|c|}{ Univariate } & \multicolumn{3}{|c|}{ Multivariate } \\
\hline & HR & $95 \% \mathrm{Cl}$ & P-value & $\mathrm{HR}$ & $95 \% \mathrm{Cl}$ & P-value \\
\hline Sex & 0.825 & $0.472-1.443$ & 0.501 & & & \\
\hline Age & 1.006 & $0.982-1.030$ & 0.626 & & & \\
\hline Tumor size (CT) & 1.045 & $0.914-1.195$ & 0.522 & & & \\
\hline Clinical TNM stage & & & 0.211 & & & \\
\hline 1 & Reference & Reference & & & & \\
\hline II & 2.840 & $0.989-8.154$ & & & & \\
\hline III & 1.591 & $0.747-3.390$ & & & & \\
\hline IV & 1.253 & $0.665-2.360$ & & & & \\
\hline Clinical Masaoka-Koga stage & & & 0.666 & & & \\
\hline 1 & Reference & Reference & & & & \\
\hline II & 0.934 & $0.358-2.432$ & & & & \\
\hline III & 1.280 & $0.657-2.494$ & & & & \\
\hline IV & 1.482 & $0.711-3.091$ & & & & \\
\hline Neoadjuvant treatment & & & & & & 0.010 \\
\hline Chemotherapy & 1.300 & $0.668-2.528$ & 0.440 & 7.202 & $1.598-32.455$ & \\
\hline Radiotherapy & 5.987 & $1.441-24.883$ & 0.014 & & & \\
\hline Concurrent chemoradiation & 0.637 & $0.088-4.620$ & 0.655 & & & \\
\hline Approach & & & 0.089 & & & 0.138 \\
\hline Sternotomy & Reference & Reference & & Reference & Reference & \\
\hline VATS & 0.565 & $0.138-2.317$ & & 0.614 & $0.140-2.689$ & \\
\hline Thoracotomy & 0.123 & $0.017-0.894$ & & 0.142 & $0.019-1.066$ & \\
\hline Extent & & & 0.8 & & & \\
\hline Thymomectomy & Reference & Reference & & & & \\
\hline Partial thymectomy & 1.149 & $0.352-3.748$ & & & & \\
\hline Total thymectomy & 1.273 & $0.620-2.615$ & & & & \\
\hline Pathologic TNM stage & & & 0.004 & & & 0.238 \\
\hline 1 & Reference & Reference & & Reference & Reference & \\
\hline II & 0.396 & $0.052-2.999$ & & 0.433 & $0.051-3.674$ & \\
\hline III & 1.609 & $0.821-3.155$ & & 1.232 & $0.497-3.053$ & \\
\hline IV & 2.981 & $1.546-5.750$ & & 2.125 & $0.832-5.429$ & \\
\hline Subtype & & & 0.743 & & & \\
\hline Squamous cell carcinoma & Reference & Reference & & & & \\
\hline Lymphoepithelioma-like & 1.590 & $0.489-5.174$ & & & & \\
\hline Sarcomatoid & 3.167 & $0.429-23.366$ & & & & \\
\hline Mucoepidermoid & 1.114 & $0.152-8.173$ & & & & \\
\hline Adenocarcinoma & 2.674 & $0.640-11.171$ & & & & \\
\hline Resection margin & & & 0.214 & & & 0.55 \\
\hline Ro & Reference & Reference & & Reference & Reference & \\
\hline $\mathrm{R} 1$ & 1.682 & $0.941-3.007$ & & 1.206 & $0.652-2.23$ & \\
\hline \multicolumn{7}{|l|}{ Adjuvant therapy (\%) } \\
\hline Chemotherapy & 1.211 & $0.681-2.153$ & 0.514 & & & \\
\hline Radiotherapy & 0.680 & $0.392-1.181$ & 0.171 & & & \\
\hline
\end{tabular}

HR, hazard ratio; $\mathrm{Cl}$, confidence interval; $\mathrm{CT}$, computed tomography; TNM, tumor-node-metastasis; VATS, video-assisted thoracoscopic surgery. 
PRECISION AND FUTURE MEDICINE

Yeong Jeong Jeon, et al.

Table 8. Cox regression analysis of prognostic factors of recurrence-free survival

\begin{tabular}{|c|c|c|c|c|c|c|}
\hline \multirow{2}{*}{ Variable } & \multicolumn{3}{|c|}{ Univariate } & \multicolumn{3}{|c|}{ Multivariate } \\
\hline & $H R$ & $95 \% \mathrm{Cl}$ & P-value & $\mathrm{HR}$ & $95 \% \mathrm{Cl}$ & P-value \\
\hline Sex & 1.035 & $0.642-1.669$ & 0.886 & & & \\
\hline Age & 0.992 & $0.972-1.013$ & 0.464 & & & \\
\hline Tumor size $(\mathrm{CT})$ & 1.071 & $0.964-1.19$ & 0.203 & & & \\
\hline Clinical TNM stage & & & 0.001 & & & 0.020 \\
\hline I & Reference & Reference & & Reference & Reference & \\
\hline ॥ & 5.092 & $2.234-11.607$ & & 3.749 & $1.418-9.912$ & \\
\hline III & 1.334 & $0.613-2.905$ & & 0.725 & $0.304-1.728$ & \\
\hline IV & 1.981 & $1.182-3.32$ & & 0.977 & $0.519-1.839$ & \\
\hline Clinical Masaoka-Koga stage & & & $<0.001$ & & & 0.245 \\
\hline I & Reference & Reference & & Reference & Reference & \\
\hline$\|$ & 2.066 & $0.797-5.357$ & & 1.452 & $0.487-4.329$ & \\
\hline III & 3.094 & $1.469-6.517$ & & 1.882 & $0.679-5.215$ & \\
\hline IV & 6.284 & $2.96-13.341$ & & 2.760 & $0.992-7.680$ & \\
\hline \multicolumn{7}{|l|}{ Neoadjuvant treatment } \\
\hline Chemotherapy & 2.027 & $1.218-3.371$ & 0.007 & 1.106 & $0.599-2.043$ & 0.748 \\
\hline Concurrent chemoradiation & 3.298 & $1.193-9.118$ & 0.021 & 3.277 & $0.955-11.248$ & 0.059 \\
\hline Approach & & & 0.147 & & & \\
\hline Sternotomy & Reference & Reference & & & & \\
\hline VATS & 0.427 & $0.171-1.062$ & & & & \\
\hline Thoracotomy & 1.296 & $0.521-3.223$ & & & & \\
\hline Extent & & & 0.764 & & & \\
\hline Thymomectomy & Reference & Reference & & & & \\
\hline Partial thymectomy & 1.407 & $0.554-3.576$ & & & & \\
\hline Total thymectomy & 1.089 & $0.582-2.039$ & & & & \\
\hline Pathologic TNM stage & & & $<0.001$ & & & 0.045 \\
\hline I & Reference & Reference & & Reference & Reference & \\
\hline II & 1.517 & $0.507-4.542$ & & 1.070 & $0.304-3.761$ & \\
\hline III & 2.411 & $1.286-4.518$ & & 1.299 & 0.549-3.075 & \\
\hline IV & 5.545 & $3.012-10.206$ & & 3.297 & $1.276-8.519$ & \\
\hline Subtype & & & 0.986 & & & \\
\hline Squamous cell carcinoma & Reference & Reference & & & & \\
\hline Lymphoepithelioma-like & 0.749 & $0.183-3.066$ & & & & \\
\hline Adenocarcinoma & 1.314 & $0.321-5.375$ & & & & \\
\hline NOS & 0.602 & $0.189-1.921$ & & & & \\
\hline Resection margin & & & 0.021 & & & 0.533 \\
\hline RO & Reference & Reference & & Reference & Reference & \\
\hline $\mathrm{R} 1$ & 1.897 & $1.1-3.27$ & & 0.819 & $0.438-1.532$ & \\
\hline Adjuvant therapy (\%) & & & & & & 0.248 \\
\hline Chemotherapy & 1.702 & $1.033-2.804$ & 0.037 & 1.386 & $0.796-2.413$ & \\
\hline Radiotherapy & 0.796 & $0.494-1.285$ & 0.351 & & & \\
\hline
\end{tabular}

HR, hazard ratio; Cl, confidence interval; CT, computed tomography; TNM, tumor-node-metastasis; VATS, video-assisted thoracoscopic surgery; NOS, not otherwise specified. 
0.138), although neoadjuvant radiotherapy was associated with poor OS (hazard ratio, 7.2; 95\% confidence interval, 1.6 to $32.46 ; \mathrm{P}=0.01$ ). The multivariable Cox regression analysis for RFS showed pathologic TNM stage was a significant prognostic factor of RFS $(P=0.045)$. However, VATS had not effect on either OR or RFS.

\section{DISCUSSION}

Thymic carcinomas are very rare, and most literatures about thymic carcinomas are retrospective, small-case, single-center studies. Only a few retrospective large-sample multicenter studies on long-term outcome of thymic carcinoma were reported in the United States (Surveillance, Epidemiology, and End Results database, $n=290$ ), Europe (European Society of Thoracic Surgeons database, n=229), Japan (Japanese Association for Research of the Thymus database, $\mathrm{n}=306$ ), and China (Chinese Alliance for Research of Thymoma database, $n=369$ ) [2,11-13]. In Korea, the KART developed a multi-institutional retrospective database in 2014, and collected the data of thymic epithelial tumor, including thymic carcinoma.

In early stage thymic tumor, surgery for complete resection has remained the mainstay of curative treatment because complete resection was a significant prognostic factor in the many studies of thymic carcinoma $[2,14]$. Traditionally, this has been conducted with open procedures, especially median sternotomy. According to the European Society of Medical Oncology (ESMO) guidelines on thymic tumors, the standard surgical approach for resectable thymic epithelial tumor remains median sternotomy (grade IV, level A) [15]. More recently, due to the widespread use of VATS, there has been a progressive adoption of these techniques in surgery for thymic carcinoma. The ESMO guidelines suggest that minimally invasive surgery is an option for early stage tumor in the hands of appropriately trained surgeons. Several advantages of VATS for the treatment of thymic tumors have been known in a number of literatures in the last decade. VATS provide non inferior oncologic outcomes than open approach and is associated with shorter length of hospital stay, reduced blood loss and duration of chest tube [9,14-17]. Unfortunately, most of these literatures are retrospective studies and no randomized clinical study has been published because of the rarity of thymic tumor.

In the present study, most patients with thymic carcinoma who underwent VATS approach had tumor with less than 5 $\mathrm{cm}$ (mean tumor size, $3.8 \pm 1.1 \mathrm{~cm}$ ) and TNM stage I (90.9\%).
Therefore, we analyzed 55 patients (28 sternotomy and 27 VATS) who had tumor with less than $5 \mathrm{~cm}$ and clinical TNM stage I according to the approach of surgery. VATS was associated with shorter operation time, lower blood loss, reduced lengths of POHS, ICU stay, and chest tube duration. Furthermore, the 5-year OS and RFS rates and pattern of recurrence were not significantly different between the groups. Therefore, VATS can be applied to patients who had thymic carcinoma with less than $5 \mathrm{~cm}$ and clinical TNM stage I.

VATS was performed mostly in patients who did not confirm histology by preoperative biopsy according to our result. The National Comprehensive Cancer Network recommends that surgical biopsy should be avoided if a resectable thymic tumor is strongly suspected based on clinical and radiologic features [18]. However, histology of the thymic tumor is difficult to distinguish by imaging in case of small tumor with no lymphadenopathy [19]. In our series, although thymic tumor revealed to be thymic carcinoma (WHO type C) intraoperatively or postoperatively, VATS can be acceptable if complete resection was achieved.

The present study has limitations. First, the number of cases is small because of the rarity of thymic carcinoma, with only 201 cases over 7 years. Second, this is retrospective study, and selection bias exists. Surgeons would preferred VATS for less invasive tumor such as small size without invasion to innominate vein or great vessels on preoperative $\mathrm{CT}$. Therefore, we analyzed subgroup who had tumor with less than $5 \mathrm{~cm}$ and TNM stage I. Third, the follow-up period after surgery is slightly insufficient. Because patients with thymic carcinoma have good prognosis, a follow-up time of 10 years or more may be necessary to identify substantial long-term outcomes. Fourth, surgical techniques, the degree of individual skills, and postoperative care of individual center could not be evaluated.

In conclusion, the VATS was applied for tumor less than 5 $\mathrm{cm}$ and TNM stage I. The VATS group had shorter duration of chest tube, mechanical ventilation, postoperative hospital day, and lower incidence of postoperative complication. The 5-year OS and RFS was not significantly different between the groups. Therefore, the VATS approach of surgical treatment for thymic carcinoma can be selectively employed in small and TNM stage I tumor without compromise of oncologic outcome.

\section{CONFLICTS OF INTEREST}

No potential conflict of interest relevant to this article was re- 
ported.

\section{ACKNOWLEDGMENTS}

Members of the KART Study Group: Sumin Shin, Jong Ho Cho, Hong Kwan Kim, Jhingook Kim, Jae II Zo, Young Mog Shim (Department of Thoracic and Cardiovascular Surgery, Samsung Medical Center, Sungkyunkwan University School of Medicine, Seoul, Korea); Geun Dong Lee, Su Kyung Hwang, Sehoon Choi, Hyeong Ryul Kim, Yong-Hee Kim, Dong Kwan Kim, Seung-Il Park (Department of Thoracic and Cardiovascular Surgery, Asan Medical Center, University of Ulsan College of Medicine, Seoul, Korea); Samina Park, Kwan Yong Hyun, Yoohwa Hwang, Hyun Joo Lee, In Kyu Park, Chang Hyun Kang, Young Tae Kim (Department of Thoracic and Cardiovascular Surgery, Seoul National University Hospital, Seoul National University College of Medicine, Seoul, Korea); and Chang Young Lee, Jin Gu Lee, Hyo Chae Paik, Dae Joon Kim, Kyung Young Chung (Department of Thoracic and Cardiovascular Surgery, Severance Hospital, Yonsei University College of Medicine, Seoul, Korea).

\section{ORCID}

Yeong Jeong Jeon

https://orcid.org/0000-0001-6745-6131

Yong Soo Choi https://orcid.org/0000-0001-8492-7644

Jong Ho Cho

Hong Kwan Kim

Geun Dong Lee https://orcid.org/0000-0003-3362-4621 https://orcid.org/0000-0002-7815-3336 https://orcid.org/0000-0003-1890-7455

Dong Kwan Kim Chang Hyun Kang Young Tae Kim Chang Young Lee Jin Gu Lee https://orcid.org/0000-0003-1984-0352 https://orcid.org/0000-0002-1612-1937 https://orcid.org/0000-0001-9006-4881 https://orcid.org/0000-0002-2404-9357 https://orcid.org/0000-0003-2767-6505

\section{AUTHOR CONTRIBUTIONS}

Conception or design: YJJ, YSC.

Acquisition, analysis, or interpretation of data: YJJ, YSC, JHC, HKK, GDL, DKK, CHK, YTK, CYL, JGL.

Drafting the work or revising: YJJ, YSC.

Final approval of the manuscript: YJJ, YSC.

\section{REFERENCES}

1. Suster S, Moran CA. Primary thymic epithelial neoplasms showing combined features of thymoma and thymic car- cinoma: a clinicopathologic study of 22 cases. Am J Surg Pathol 1996;20:1469-80.

2. Weksler B, Dhupar R, Parikh V, Nason KS, Pennathur A, Ferson PF. Thymic carcinoma: a multivariate analysis of factors predictive of survival in 290 patients. Ann Thorac Surg 2013;95:299-303.

3. Shepherd A, Riely G, Detterbeck F, Simone CB 2nd, Ahmad $U$, Huang J, et al. Thymic carcinoma management patterns among International Thymic Malignancy Interest Group (ITMIG) physicians with consensus from the Thymic Carcinoma Working Group. J Thorac Oncol 2017; 12:745-51.

4. Zhai Y, Hui Z, Ji W, Wang X, Liang J, Mao Y, et al. A single-center analysis of the treatment and prognosis of patients with thymic carcinoma. Ann Thorac Surg 2017;104: 1718-24.

5. Jurado J, Javidfar J, Newmark A, Lavelle M, Bacchetta M, Gorenstein L, et al. Minimally invasive thymectomy and open thymectomy: outcome analysis of 263 patients. Ann Thorac Surg 2012;94:974-81.

6. Odaka M, Akiba T, Mori S, Asano H, Marushima H, Yamashita M, et al. Oncological outcomes of thoracoscopic thymectomy for the treatment of stages I-III thymomas. Interact Cardiovasc Thorac Surg 2013;17:285-90.

7. Ye B, Tantai JC, Ge XX, Li W, Feng J, Cheng M, et al. Surgical techniques for early-stage thymoma: video-assisted thoracoscopic thymectomy versus transsternal thymectomy. J Thorac Cardiovasc Surg 2014;147:1599-603.

8. Chao YK, Liu YH, Hsieh MJ, Wu YC, Chen TP, Lu MS, et al. Long-term outcomes after thoracoscopic resection of stage I and II thymoma: a propensity-matched study. Ann Surg Oncol 2015;22:1371-6.

9. Friedant AJ, Handorf EA, Su S, Scott WJ. Minimally invasive versus open thymectomy for thymic malignancies: systematic review and meta-analysis. J Thorac Oncol 2016; 11:30-8.

10. Ruffini E, Filosso PL, Guerrera F, Lausi P, Lyberis P, Oliaro A. Optimal surgical approach to thymic malignancies: new trends challenging old dogmas. Lung Cancer 2018; 118:161-70.

11. Ruffini E, Detterbeck F, Van Raemdonck D, Rocco G, Thomas P, Weder W, et al. Thymic carcinoma: a cohort study of patients from the European society of thoracic surgeons database. J Thorac Oncol 2014;9:541-8.

12. Hishida T, Nomura S, Yano M, Asamura H, Yamashita M, Ohde $\mathrm{Y}$, et al. Long-term outcome and prognostic factors of surgically treated thymic carcinoma: results of 306 
cases from a Japanese Nationwide Database Study. Eur J Cardiothorac Surg 2016;49:835-41.

13. Fu H, Gu ZT, Fang WT, Fu JH, Shen Y, Han YT, et al. Longterm survival after surgical treatment of thymic carcinoma: a retrospective analysis from the Chinese alliance for research of thymoma database. Ann Surg Oncol 2016; 23:619-25.

14. Ahmad U, Yao X, Detterbeck F, Huang J, Antonicelli A, Filosso PL, et al. Thymic carcinoma outcomes and prognosis: results of an international analysis. J Thorac Cardiovasc Surg 2015;149:95-100.

15. Girard N, Ruffini E, Marx A, Faivre-Finn C, Peters S; ESMO Guidelines Committee. Thymic epithelial tumours: ESMO clinical practice guidelines for diagnosis, treatment and follow-up. Ann Oncol 2015;26 Suppl 5:v40-55.

16. Hess NR, Sarkaria IS, Pennathur A, Levy RM, Christie NA, Luketich JD. Minimally invasive versus open thymecto- my: a systematic review of surgical techniques, patient demographics, and perioperative outcomes. Ann Cardiothorac Surg 2016;5:1-9.

17. Wang H, Gu Z, Ding J, Tan L, Fu J, Shen Y, et al. Perioperative outcomes and long-term survival in clinically early-stage thymic malignancies: video-assisted thoracoscopic thymectomy versus open approaches. J Thorac Dis 2016;8:673-9.

18. Ettinger DS, Riely GJ, Akerley W, Borghaei H, Chang AC, Cheney RT, et al. Thymomas and thymic carcinomas: clinical practice guidelines in oncology. J Natl Compr Canc Netw 2013;11:562-76.

19. Han J, Lee KS, Yi CA, Kim TS, Shim YM, Kim J, et al. Thymic epithelial tumors classified according to a newly established WHO scheme: CT and MR findings. Korean J Radiol 2003;4:46-53. 